\title{
Fault Diagnosis of Analog Circuits Based on CFNN
}

\author{
Meirong $\mathrm{Liu}^{1,2, \mathrm{a}}$, Yigang $\mathrm{He}^{3, \mathrm{~b}}$, Xiangxin $\mathrm{Li}^{4, \mathrm{c}}$ \\ ${ }^{1}$ College of Physics and Information Science, Hunan Normal University, Changsha, 410081, China \\ ${ }^{2}$ Electric Engineering Postdoctoral Center, Hefei University of Technology, Hefei, 230009, China \\ ${ }^{3}$ College of Electrical and Automation Engineering, Hefei University of Technology, Hefei, 230009, \\ China \\ ${ }^{4}$ College of Electronical and Information Engineering, Changsha University of Science and \\ Technology, Changsha, 410004, China

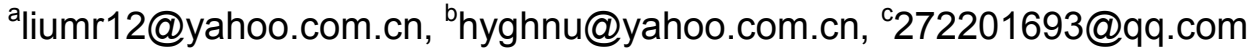

Keywords: analog circuit; chaos; fuzzy; neural network

\begin{abstract}
An analog circuits fault diagnosis method based on chaotic fuzzy neural network (CFNN) is presented. The method uses the advantage of the global movement characteristic inherent in chaos to overcome the shortcomings that BPNN is usually trapped to a local optimum and it has a low speed of convergence weights. The chaotic mapping was added into BPNN algorithm, and the initial value of the network was selected. The algorithm can effectively and reliably be used in analog circuit fault diagnosis by comparing the two methods and analyzing the results of the example.
\end{abstract}

\section{Introduction}

From the 1960's, lots of approaches and theories are presented in analog circuit fault diagnosis ${ }^{[1-5]}$. But there are many difficulties in analog circuit fault diagnosis because of its continuous input and output response, the tolerance of the electric element and the non-linearity that commonly existed in analog circuit. With the development of digital-analog circuit and the VLSI technology, the analog circuit test and the fault diagnosis are urgently demanded.

The artificial neural network (ANN) has many virtues, such as association memory ability, strong robustness and non-linearity. It is applied widely in various fields. BPNN is one of the most extensive application networks. But the training processes of neural networks can be easily entrapped into the local minima and slow convergence.

Since 1965, L.A. Zadeh put forward fuzzy theories, and relevant theories and application of the fuzzy information all make important progress. The fuzzy logic has the abstract thinking emulational characteristic of human brain, and the neural network has image thinking of human brain. Currently, it is an important form for realization of the intelligence diagnosis that the fuzzy logic and the neural network are combined to imitate human brain in terms of both the abstract thinking and the image thinking.

Chaos is a kind of widespread phenomenon for nonlinear dynamics system. It has special dynamics property: regularity, random and ergodicity.

In this paper, the method of chaotic fuzzy neural networks is proposed for diagnosing faulty circuits for their quick convergence and effective classification. Chaos variables are applied in searching for neural network structure, in which node numbers of hidden layer and all weight parameters of neural network are in chaotic state. All neural network structure is protean. Compared with the ordinary neural network, the rate of accuracy in analog circuit fault diagnosis can be further enhanced when the tolerance is included; moreover, the diagnosis time has also been further reduced.

\section{Fuzzy neural network}

The fuzzy neural network (FNN) is a kind of new neural network; it is the neural network that the fuzzy method or the fuzzy weight coefficient is leaded into the network. 
Supposes the fuzzy rule of input and output relations is

$R_{J}:$ if $x_{1}$ is $A^{j 1}{ }_{1}$ and $x 2$ is $A^{j 2}{ }_{2}$ and $\ldots$ and $x_{n}$ is $A^{j n}{ }_{n}$ then $y$ is $B^{j} . j=1,2, \ldots, m ; n$ expresses the rule total; $j_{i} \in\left\{1,2, \cdots, m_{i}\right\}, m_{i}$ is fuzzy graduation number.

The inputs of neural network use the fuzzy method of the simple-point fuzzy set; the minimum computation is used to express the fuzzy reasoning; clear computation uses the weighted mean; the Gaussian function is used to express the degree of membership, then the relations of the fuzzy system input and output is

$$
y=\left.u\right|_{n}=\frac{\sum_{j=1}^{m} W^{J}\left\{\prod_{i=1}^{n} \exp \left[-\left(\frac{x_{i}-a_{i}^{j i}}{b_{i}^{j i}}\right)^{2}\right]\right\}}{\sum_{j=1}^{m}\left\{\prod_{i=1}^{n} \exp \left[-\left(\frac{x_{i}-\mu_{i}^{j i}}{\delta_{i}^{j i}}\right)^{2}\right]\right\}}
$$

In which $W^{J}, a_{i}^{j i}, b_{i}^{j i}$ respectively are consequent network connection weight, fuzzy set center value and width of antecedent variable $\mathrm{x}_{\mathrm{i}}$.

The FNN structure is shown in Fig.1.

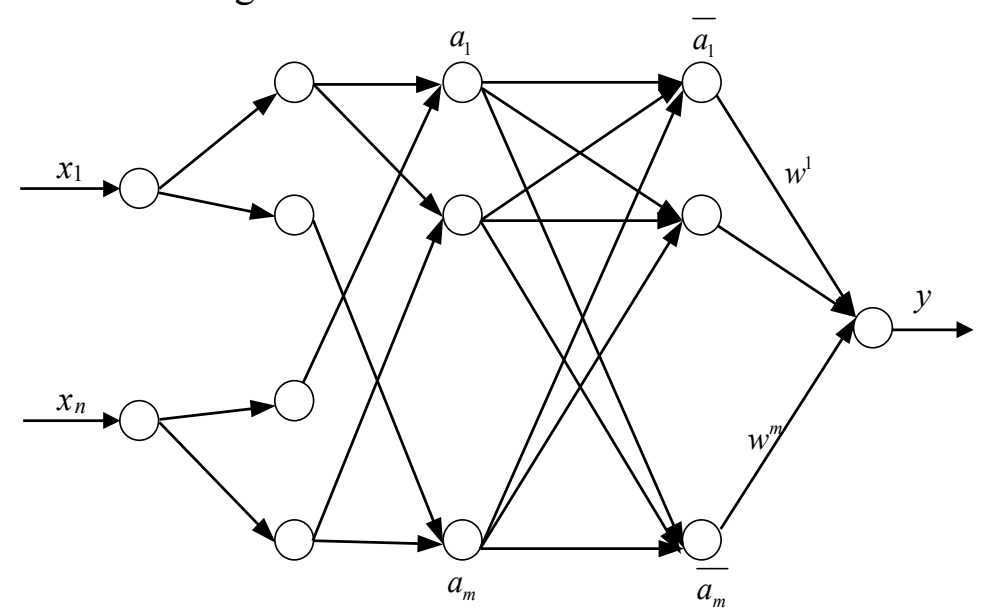

Fig. 1 Fuzzy neural network structure

In Fig. 1, the first layer is the input level, the second layer is the computation of the degree of member-ship function; the third layer is used to match the fuzzy rule antecedent, and calculate triggering intensity of each regular; the fourth layer is used to compute normalized value; the last layer is used to clarify the computation.

$$
\begin{gathered}
\mu_{i}^{j i}=\exp \left[-\left(\frac{x_{i}-a_{i}^{j i}}{b_{i}^{j i}}\right)^{2}\right] \\
a_{j}=\prod_{i=1}^{n} \mu_{i}^{j i}=\prod_{i=1}^{n} \exp \left[-\left(\frac{x_{i}-a_{i}^{j i}}{b_{i}^{j i}}\right)^{2}\right] \\
\overline{a_{j}}=\frac{a_{j}}{\sum_{j=1}^{m} a_{j}} \\
y=u_{n}=\sum_{j=1}^{m} W^{J} \overline{a_{j}}
\end{gathered}
$$

The study signal is defined:

$$
J=\frac{1}{2}\left(u_{r}-u_{n}\right)^{2}
$$


If the general BP algorithm is used, the study algorithm of its parameter $W^{J}, a_{i}^{j i}, b_{i}^{j i}$ is defined

$$
W^{J}(k+1)=W^{J}(k)+\Delta W^{J}(k)
$$

Where $\Delta W^{J}(k)=-\eta \frac{\partial J}{\partial W^{J}}$.

$$
a_{i}^{j i}(k+1)=a_{i}^{j i}(k)+\Delta a_{i}^{j i}(k)
$$

Where $\Delta a_{i}^{j i}(k)=-\eta \frac{\partial J}{\partial a_{i}^{j i}}$.

$$
b_{i}^{j i}(k+1)=b_{i}^{j i}(k)+\Delta b_{i}^{j i}(k)
$$

Where $\Delta b_{i}^{j i}(k)=-\eta \frac{\partial J}{\partial b_{i}^{j i}}$.

\section{Fuzzy neural network based on chaos theory}

The nonlinear self-feedback of weight increase: $h(x)=\sqrt{e} \frac{A}{R} x e^{-x^{2} / R^{2}}$ is introduced, and x separately equal $\Delta W^{J}(k), \Delta a_{i}^{j i}(k), \Delta b_{i}^{j i}(k)$ in the Eq.7, Eq. 8 and Eq. 9. Then the Eq.(7-9) correspondingly become the following three equations.

$$
\begin{aligned}
& W^{J}(k+1)=W^{J}(k)-\eta \frac{\partial J}{\partial W^{J}}+h\left(\Delta W^{J}(k)\right) \\
& a_{i}^{j i}(k+1)=a_{i}^{j i}(k)-\eta \frac{\partial J}{\partial a_{i}^{j i}}+h\left(\Delta a_{i}^{j i}(k)\right) \\
& b_{i}^{j i}(k+1)=b_{i}^{j i}(k)-\eta \frac{\partial J}{\partial b_{i}^{j i}}+h\left(\Delta b_{i}^{j i}(k)\right)
\end{aligned}
$$

Where $A$ and $R$ respectively denote the speed and the radius of nonlinear self-feedback. They can control the sphere of the weight. If $R$ is invariable, $A$ will decide the power which shifts between the energy minimum of the weight dynamics system. The bigger $A$ is, the bigger the scope of the weight revises is. The $|x|$ denotes the speed that dynamics system approaches to the fixed point. If $|x|$ is very big, then the system is far away the fixed point, $h(x)$ should reduce rapidly in order to rapidly approach system fixed point. When $|x|$ is at the intermediate quantity, the weight dynamics system will enter some nearby domain of the fixed point, and the self-feedback function of $h(x)$ will have the new driving influence to jump out of the fixed point, finally it will cause the weight to enter some domain of the most superior fixed point under the overall significance.

\section{Simulation}

Diagnosis circuit shows in Fig. $2^{[6]}$, the unit of resistance and capacitance separately is $\mathrm{k} \Omega$ and $\mathrm{uF}$. In the Example, the simulation is carried on under the direct-current condition, and only the tolerance of the resistance is taken into consideration; the tolerance of the resistances is $-5 \% \sim 5 \%$. The test node are showed in Fig.2(node 1, 2, 3, 4, 5, 6). So the test vector is six dimensions. 


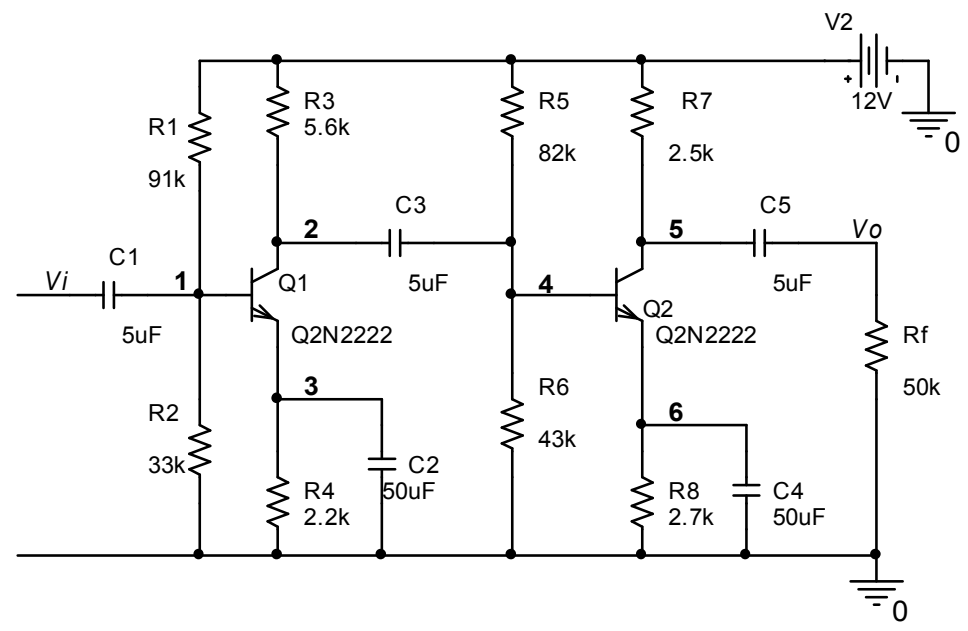

Fig. 2 Analog circuit

Suppose B, C, E separately denotes base, collector and emitter of transistor, OC and SC separately denote open circuit and short circuit. Fault muster as follows: F0:Normal, F1:Q1BES, F2:Q1CES, F3:Q1BCS, F4:Q2BES, F5:Q2CES, F6:Q2BCS, F7:C2SC, F8:C3SC, F9:C4SC, F10:R1SC, F11:R3SC, F12:R3OC, F13:R4OC, F14:R5SC, F15:R7SC, F16:R8OC

Circuit is simulated with PSpice, each test node voltage values is shown in Table 1. The $V_{1}, V_{2}$, $\mathrm{V}_{3}, \mathrm{~V}_{4}, \mathrm{~V}_{5}$ and $\mathrm{V}_{6}$ separately denote measurement voltage of test node. It is obvious that fault sample value is very different in Table 1 . So Fault samples are preprocessed before they are trained in the neural network. The processed data will be used to train and test the neural network.

Table 1 Test nodes voltage of circuit fault states [V]

\begin{tabular}{lllllll}
\hline state & $\mathrm{V}_{1}$ & $\mathrm{~V}_{2}$ & $\mathrm{~V}_{4}$ & $\mathrm{~V}_{5}$ & $\mathrm{~V}_{6}$ & $\mathrm{~V}_{3}$ \\
\hline F0 & 3.025 & 5.986 & 3.916 & 8.994 & 3.267 & 2.378 \\
F1 & 0.266 & 12.00 & 3.916 & 8.994 & 3.267 & 0.266 \\
F2 & 3.194 & 3.385 & 3.916 & 8.994 & 3.267 & 3.385 \\
F3 & 3.815 & 3.815 & 3.916 & 8.994 & 3.267 & 3.159 \\
F4 & 3.025 & 5.986 & 0.361 & 12.00 & 0.361 & 2.378 \\
F5 & 3.025 & 5.986 & 4.128 & 6.231 & 6.231 & 2.378 \\
F6 & 3.025 & 5.986 & 6.444 & 6.444 & 5.779 & 2.378 \\
F7 & 0.673 & 0.045 & 3.916 & 8.994 & 3.267 & 0 \\
F8 & 3.024 & 5.626 & 5.626 & 7.431 & 4.965 & 2.377 \\
F9 & 3.025 & 5.986 & 0.691 & 0.065 & 0 & 2.378 \\
F10 & 12 & 11.22 & 3.916 & 8.994 & 3.267 & 11.21 \\
F11 & 3.035 & 12.00 & 3.916 & 8.994 & 3.267 & 2.39 \\
F12 & 0.849 & 0.217 & 3.916 & 8.994 & 3.267 & 0.213 \\
F13 & 3.194 & 12.00 & 3.916 & 8.994 & 3.267 & 11.96 \\
F14 & 3.025 & 5.986 & 12.00 & 11.24 & 11.23 & 2.378 \\
F15 & 3.025 & 5.986 & 3.922 & 12 & 3.274 & 2.378 \\
F16 & 3.025 & 5.986 & 4.128 & 12 & 11.96 & 2.378 \\
\hline
\end{tabular}

In the example, the neural network is designed 6 input neurons and 5 output neurons. Each state corresponding to the binary output is shown in Table 2.

Table 2 The binary output of each state

\begin{tabular}{cccccc}
\hline state & code & state & code & state & code \\
\hline F0 & 00000 & F1 & 00001 & F2 & 00010 \\
F3 & 00011 & F4 & 00100 & F5 & 00101 \\
F6 & 00110 & F7 & 00111 & F8 & 01000 \\
F9 & 01001 & F10 & 01010 & F11 & 01011 \\
F12 & 01100 & F13 & 01101 & F14 & 01110 \\
F15 & 01111 & F16 & 10000 & & \\
\hline
\end{tabular}


The CFNN algorithm and the BPNN are separately used to test and diagnose the circuit; their training error goal curves are shown in Fig.3, 3(a) is the CFNN algorithm training goal curve, chart $3(b)$ is the BPNN algorithm training goal curve. When simulation algorithm err_goal $=0.0001$.

In Fig.3, CFNN algorithm has converged with 276 steps; But the BPNN algorithm has converged with 564 steps to get the anticipated result. Based on the neural networks trained with the chaos and fuzzy, the fault classes can correctly be classified for at least $98 \%$ of the test data of our example circuits. But based on the BPNN trained, the fault classes can correctly be classified for at least $78 \%{ }^{[6]}$. It is obvious that the convergence rate and time of CFNN are quicker than the ordinary BPNN. Thus the chaotic fuzzy neural network method strengthens the timeliness and the accuracy of fault diagnosis system.

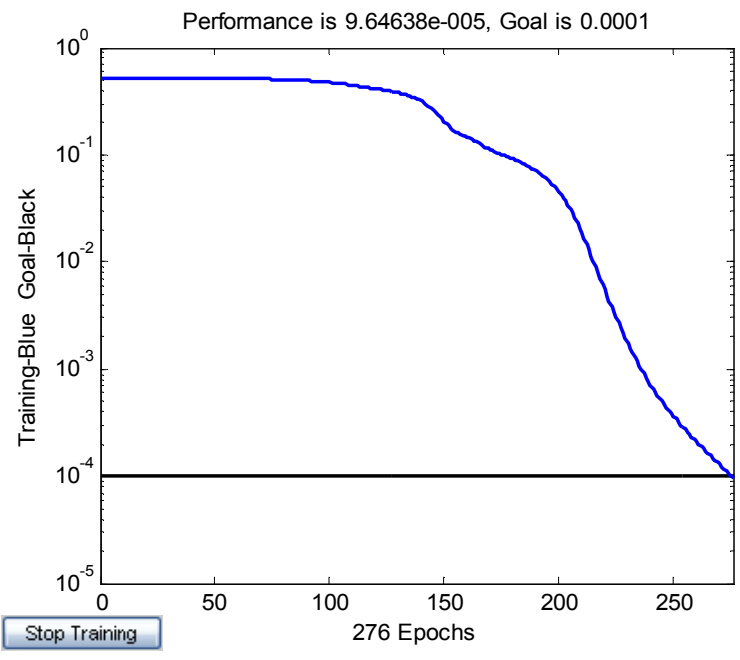

(a) CFNN algorithm training error goal curve

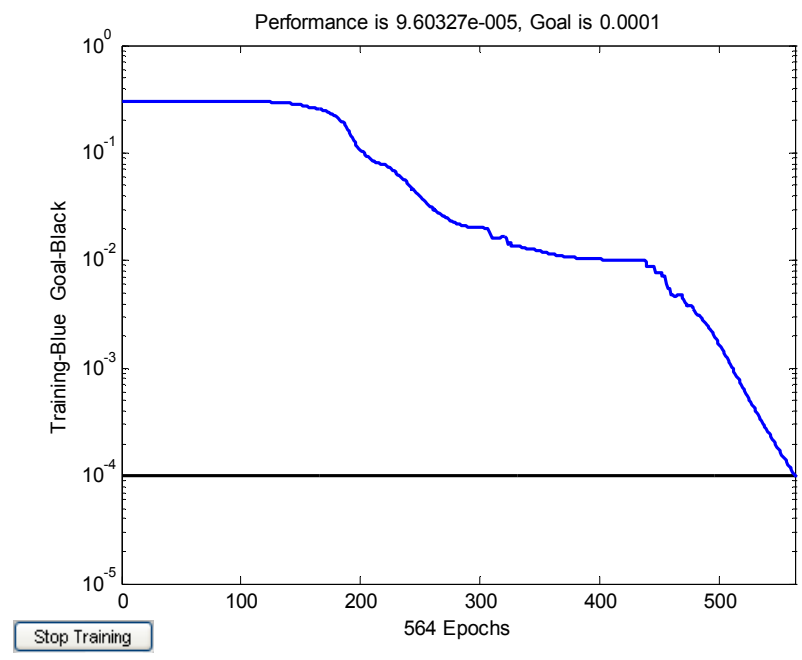

(b) BPNN algorithm training error goal curve

Fig. 3 Training error goal curve

\section{Conclusions}

A new method of analog circuit fault diagnosis based on CFNN is proposed in this paper. The method can automatically classify the test data, and structure fuzzy neural network to diagnose the fault of analog circuit. The method is proved effective by the experiment. The accuracy rate and speed of fault diagnosis have been further enhanced compared with the traditional diagnosis method. The method can also be applied to other domains in which the fuzzy logic, neural network and chaos are combined. More importantly, if these input feature vectors are not overlapped in the circuits, the method can diagnose not only soft faults but also hard faults.

\section{Acknowledgement}

This work was supported by the National Natural Science Funds of China for Distinguished Young Scholar under Grant (No. 50925727), National Natural Science Foundation of China under Grant (No.60876022), Scientific Research Fund of Hunan Provincial Education Department(No. 11C0800). 


\section{Reference}

[1] Yang Shiyuan, analog system fault diagnosis and reliability design [M]. Qinghua University publishing company, Beijin, 1993.

[2] Lifen Yuan, Yigang He, Jiaoying Huang etal.. A New Neural-Network-Based Fault Diagnosis Approach for Analog Circuits by Using Kurtosis and Entropy as a Preprocessor[J]. IEEE Transactions on Instrumentation and Measurement, 2010, 59(3): 586-595

[3] Aminian M, Aminian F. A modular fault- diagnostic system for analog electronic circuits using neural networks with wavelet transform as a preprocessor[J]. IEEE Transactions on Instrumentation and Measurement, 2007, 56(5): 1546-1554.

[4] Wang Peng, Yang Shiyuan. A new diagnosis approach for handling tolerance in analog and mixed-signal circuits by using fuzzy math[J]. IEEE Transactions on CAS I , 2005, 52(10): 2118-2127.

[5] Y. He, Y. Tan, Y. Sun. Wavelet Neural Network Approach for Fault Diagnosis of Analogue Circuits[J]. IEE Proc. Circuits Devices Syst., 2004,151,(4), pp.379-384.

[6] Liu Meirong. Analog circuit fault diagnosis based on GA, wavelet and neural network [D]. Hunan University, Changsha, 2009: 22-25 